\title{
Expansion of Zirconium Oxide Clusters by 3d/4f Ions
}

Sebastian Schmitz, ${ }^{a, b}$ Natalya V. Izarova, ${ }^{a, c}$ Jan van Leusen, ${ }^{a}$ Kevin Kleemann, ${ }^{a}$ Kirill Yu. Monakhov ${ }^{b, *}$ and Paul Kögerler ${ }^{a, c, *}$

${ }^{a}$ Institut für Anorganische Chemie, RWTH Aachen University, Landoltweg 1, 52074 Aachen, Germany.E-Mail: paul.koegerler@ac.rwth-aachen.de

${ }^{b}$ Leibniz Institute of Surface Engineering (IOM), Permoserstraße 15, 04318 Leipzig, Germany. E-Mail: kirill.monakhov@iom-leipzig.de

${ }^{c}$ Jülich-Aachen Research Alliance (JARA-FIT) and Peter Grünberg Institute (PGI-6), Forschungszentrum Jülich, 52425 Jülich, Germany. 
Table S1. Crystal data and structure refinement details for compounds $1-4$

\begin{tabular}{|c|c|c|c|c|}
\hline Sample & 1 & 2 & 3 & 4 \\
\hline Empirical formula & $\begin{array}{c}\mathrm{C}_{74} \mathrm{H}_{135} \mathrm{Fe}_{2} \mathrm{La}_{2^{-}} \\
\mathrm{N}_{5} \mathrm{O}_{46} \mathrm{Zr}_{6}\end{array}$ & $\begin{array}{c}\mathrm{C}_{72} \mathrm{H}_{132} \mathrm{Ce}_{2} \mathrm{Fe}_{2^{-}} \\
\mathrm{N}_{4} \mathrm{O}_{46} \mathrm{Zr}_{6}\end{array}$ & $\begin{array}{c}\mathrm{C}_{72} \mathrm{H}_{132} \mathrm{Fe}_{2} \mathrm{~N}_{4^{-}} \\
\mathrm{O}_{46} \mathrm{Pr}_{2} \mathrm{Zr}_{6}\end{array}$ & $\begin{array}{c}\mathrm{C}_{72} \mathrm{H}_{132} \mathrm{Fe}_{2} \mathrm{~N}_{4^{-}} \\
\mathrm{Nd}_{2} \mathrm{O}_{46} \mathrm{Zr}_{6}\end{array}$ \\
\hline $\begin{array}{l}\text { Formula weight /g } \\
\text { mol }^{-1}\end{array}$ & 2767.70 & 2729.07 & 2730.65 & 2737.31 \\
\hline Crystal system & Monoclinic & Monoclinic & Monoclinic & Monoclinic \\
\hline Space group & $P 2_{1} / n$ & $P 2_{1} / n$ & $P 2_{1} / n$ & $P 2_{1} / n$ \\
\hline$a / \AA$ & $14.4632(17)$ & $16.4963(10)$ & $16.506(2)$ & $16.459(3)$ \\
\hline$b / \AA$ & $18.030(2)$ & $16.4593(10)$ & $16.478(2)$ & $16.517(3)$ \\
\hline$c / \AA$ & $20.495(2)$ & $19.0501(11)$ & $19.005(3)$ & $19.032(4)$ \\
\hline$\beta$ & $93.170(2)^{\circ}$ & $94.1590(10)^{\circ}$ & $94.173(3)^{\circ}$ & $94.27(3)^{\circ}$ \\
\hline Volume / $\AA^{3}$ & $5336.5(11)$ & $5158.8(5)$ & $5155.5(12)$ & $5159.4(18)$ \\
\hline$Z$ & 2 & 2 & 2 & 2 \\
\hline$D_{\text {calc }} / \mathrm{g} \mathrm{cm}^{-3}$ & 1.722 & 1.757 & 1.759 & 1.762 \\
\hline $\begin{array}{l}\text { Absorption } \\
\text { coefficient } / \mathrm{mm}^{-1}\end{array}$ & 1.691 & 1.802 & 1.865 & 1.926 \\
\hline$F(000)$ & 2776 & 2736 & 2740 & 2744 \\
\hline Crystal size $/ \mathrm{mm}^{3}$ & $\begin{array}{c}0.12 \times 0.14 \times \\
0.14\end{array}$ & $\begin{array}{c}0.15 \times 0.18 \times \\
0.25\end{array}$ & $\begin{array}{c}0.065 \times 0.19 \times \\
0.70\end{array}$ & $\begin{array}{c}0.21 \times 0.28 \times \\
0.36\end{array}$ \\
\hline $\begin{array}{l}\text { Theta range for } \\
\text { data collection }\end{array}$ & $1.51^{\circ}-25.12^{\circ}$ & $2.01^{\circ}-25.24^{\circ}$ & $3.16^{\circ}-27.88^{\circ}$ & $4.11^{\circ}-27.88^{\circ}$ \\
\hline $\begin{array}{l}\text { Completeness to } \\
\Theta_{\max }\end{array}$ & $99.8 \%$ & $99.9 \%$ & $99.8 \%$ & $99.5 \%$ \\
\hline Index ranges & $-17 \leq h \leq 17$ & $-23 \leq h \leq 22$ & $-21 \leq h \leq 21$ & $-21 \leq h \leq 20$ \\
\hline
\end{tabular}




\begin{tabular}{|c|c|c|c|c|}
\hline & $\begin{array}{l}-21 \leq k \leq 21 \\
-24 \leq 1 \leq 24\end{array}$ & $\begin{array}{l}-23 \leq k \leq 22 \\
-27 \leq 1 \leq 27\end{array}$ & $\begin{array}{l}-21 \leq k \leq 21 \\
-25 \leq 1 \leq 25\end{array}$ & $\begin{array}{l}-21 \leq k \leq 21 \\
-19 \leq 1 \leq 25\end{array}$ \\
\hline $\begin{array}{l}\text { Reflections } \\
\text { collected }\end{array}$ & 56466 & 76754 & 144473 & 74244 \\
\hline $\begin{array}{l}\text { Independent } \\
\text { reflections }\end{array}$ & 9529 & 15299 & 12279 & 12265 \\
\hline$R_{\mathrm{int}}$ & 0.0917 & 0.0948 & 0.0465 & 0.0575 \\
\hline $\begin{array}{l}\text { Observed } \\
(I>2 \sigma(I))\end{array}$ & 7299 & 11964 & 10645 & 9942 \\
\hline $\begin{array}{l}\text { Absorption } \\
\text { correction }\end{array}$ & \multicolumn{3}{|c|}{ Semi-empirical from equivalents } & $\begin{array}{l}\text { Gaussian } \\
\text { integration }\end{array}$ \\
\hline$T_{\min } / T_{\max }$ & $0.5884 / 0.7452$ & $0.5720 / 0.7461$ & $0.0982 / 0.1463$ & $0.7572 / 0.8577$ \\
\hline $\begin{array}{l}\text { Data / restraints / } \\
\text { parameters }\end{array}$ & 9529 / 67 / 611 & 15299 / 66 / 596 & 12279 / 75 / 599 & 12265 / 93 / 600 \\
\hline $\begin{array}{l}\text { Goodness-of-fit } \\
\text { on } F^{2}\end{array}$ & 1.095 & 1.004 & 1.115 & 1.008 \\
\hline $\begin{array}{l}R_{1}, \quad W R_{2} \quad(I> \\
2 \sigma(I))\end{array}$ & $\begin{array}{l}R_{1}=0.0552, \\
W R_{2}=0.1384\end{array}$ & $\begin{array}{c}R_{1}=0.0367 \\
W R_{2}=0.0899\end{array}$ & $\begin{array}{c}R_{1}=0.0310 \\
W R_{2}=0.0752\end{array}$ & $\begin{array}{c}R_{1}=0.0739 \\
W R_{2}=0.1879\end{array}$ \\
\hline$R_{1}, w R_{2}$ (all data) & $\begin{array}{c}R_{1}=0.0786, \\
W R_{2}=0.1533\end{array}$ & $\begin{array}{c}R_{1}=0.0506 \\
W R_{2}=0.0964\end{array}$ & $\begin{array}{c}R_{1}=0.0395 \\
W R_{2}=0.0823\end{array}$ & $\begin{array}{c}R_{1}=0.0837 \\
W R_{2}=0.1958\end{array}$ \\
\hline $\begin{array}{l}\text { Largest diff. peak } \\
\text { and hole / e } \AA^{-3}\end{array}$ & $2.318 /-1.160$ & $2.681 /-1.605$ & $2.155 /-1.392$ & $3.305 /-2.758$ \\
\hline
\end{tabular}


Table S2. Crystal data and structure refinement details for compounds 5 - 8

\begin{tabular}{|c|c|c|c|c|}
\hline Sample & 5 & 6 & 7 & 8 \\
\hline Empirical formula & $\begin{array}{c}\mathrm{C}_{69} \mathrm{H}_{124.5} \mathrm{Fe}_{2} \mathrm{La}_{2^{-}} \\
\mathrm{N}_{5.5} \mathrm{O}_{46} \mathrm{Zr}_{6}\end{array}$ & $\begin{array}{l}\mathrm{C}_{68.5} \mathrm{H}_{123.75} \mathrm{Ce}_{2^{-}} \\
\mathrm{Fe}_{2} \mathrm{~N}_{5.25} \mathrm{O}_{46} \mathrm{Zr}_{6}\end{array}$ & $\begin{array}{l}\mathrm{C}_{68.5} \mathrm{H}_{123.75} \mathrm{Fe}_{2^{-}} \\
\mathrm{N}_{5.25} \mathrm{O}_{46} \mathrm{Pr}_{2} \mathrm{Zr}_{6}\end{array}$ & $\begin{array}{l}\mathrm{C}_{69} \mathrm{H}_{124.5} \mathrm{Fe}_{2^{-}} \\
\mathrm{N}_{5.5} \mathrm{Nd}_{2} \mathrm{O}_{46} \mathrm{Zr}_{6}\end{array}$ \\
\hline $\begin{array}{l}\text { Formula weight / } \mathrm{g} \\
\mathrm{mol}^{-1}\end{array}$ & 2704.07 & 2696.23 & 2697.81 & 2714.73 \\
\hline Crystal system & Triclinic & Triclinic & Triclinic & Triclinic \\
\hline Space group & $P-1$ & $P-1$ & $P-1$ & $P-1$ \\
\hline$a / \AA$ & $15.1564(10)$ & $15.1391(16)$ & $15.1294(9)$ & $15.135(2)$ \\
\hline$b / \AA$ & $15.8099(10)$ & $15.7687(16)$ & $15.8097(10)$ & $15.834(3)$ \\
\hline$c / \AA$ & $21.3445(14)$ & $21.361(2)$ & $21.2687(13)$ & $21.227(3)$ \\
\hline$\alpha$ & $92.9270(10)^{\circ}$ & $92.752(2)^{\circ}$ & $92.6380(10)^{\circ}$ & $87.358(2)^{\circ}$ \\
\hline$\beta$ & $91.7320(10)^{\circ}$ & $91.737(2)^{\circ}$ & $92.1280(10)^{\circ}$ & $87.882(2)^{\circ}$ \\
\hline$\gamma$ & $90.6500(10)^{\circ}$ & $90.585(2)^{\circ}$ & $90.2130(10)^{\circ}$ & $89.911(3)^{\circ}$ \\
\hline Volume / $\AA^{3}$ & $5105.2(6)$ & $5090.9(9)$ & $5078.3(5)$ & $5078.4(14)$ \\
\hline$Z$ & 2 & 2 & 2 & 2 \\
\hline$D_{\text {calc }} / \mathrm{g} \mathrm{cm}^{-3}$ & 1.759 & 1.759 & 1.764 & 1.775 \\
\hline $\begin{array}{l}\text { Absorption } \\
\text { coefficient } / \mathrm{mm}^{-1}\end{array}$ & 1.766 & 1.825 & 1.893 & 1.956 \\
\hline$F(000)$ & 2702 & 2695 & 2699 & 2714 \\
\hline Crystal size / mm³ & $\begin{array}{c}0.10 \times 0.16 \times \\
0.31\end{array}$ & $\begin{array}{c}0.10 \times 0.14 \times \\
0.18\end{array}$ & $\begin{array}{c}0.14 \times 0.19 \times \\
0.34\end{array}$ & $\begin{array}{c}0.11 \times 0.17 \times \\
0.21\end{array}$ \\
\hline $\begin{array}{l}\text { Theta range for } \\
\text { data collection }\end{array}$ & $3.19^{\circ}-26.37^{\circ}$ & $2.93^{\circ}-25.03^{\circ}$ & $3.11^{\circ}-25.24^{\circ}$ & $3.14^{\circ}-27.10^{\circ}$ \\
\hline
\end{tabular}




\begin{tabular}{|c|c|c|c|c|}
\hline $\begin{array}{l}\text { Completeness to } \\
\Theta_{\max }\end{array}$ & $99.8 \%$ & $99.6 \%$ & $99.8 \%$ & $99.7 \%$ \\
\hline Index ranges & $\begin{array}{c}-18 \leq h \leq 18 \\
-19 \leq k \leq 19 \\
-26 \leq 1 \leq 26\end{array}$ & $\begin{array}{c}-18 \leq h \leq 18, \\
-18 \leq k \leq 18, \\
-25 \leq 1 \leq 25\end{array}$ & $\begin{array}{l}-18 \leq h \leq 18, \\
-19 \leq k \leq 19, \\
-25 \leq 1 \leq 25\end{array}$ & $\begin{aligned}-19 & \leq h \leq 19, \\
-20 & \leq k \leq 20, \\
-27 & \leq 1 \leq 27\end{aligned}$ \\
\hline $\begin{array}{l}\text { Reflections } \\
\text { collected }\end{array}$ & 61532 & 55356 & 57761 & 64640 \\
\hline $\begin{array}{l}\text { Independent } \\
\text { reflections }\end{array}$ & 20817 & 17917 & 19272 & 22331 \\
\hline$R_{\text {int }}$ & 0.1248 & 0.1358 & 0.1681 & 0.1145 \\
\hline $\begin{array}{l}\text { Observed } \\
(I>2 \sigma(I))\end{array}$ & 16201 & 12086 & 15664 & 16829 \\
\hline $\begin{array}{l}\text { Absorption } \\
\text { correction }\end{array}$ & \multicolumn{4}{|c|}{ Semi-empirical from equivalents } \\
\hline$T_{\min } / T_{\max }$ & $0.5526 / 0.7461$ & $0.6462 / 0.7455$ & $\begin{array}{c}0.5507 / \\
0.7461\end{array}$ & $\begin{array}{c}0.6127 / \\
0.7461\end{array}$ \\
\hline $\begin{array}{l}\text { Data / restraints / } \\
\text { parameters }\end{array}$ & $\begin{array}{c}20817 \text { / } 90 / \\
1192\end{array}$ & 17917 / 66 / 1161 & $\begin{array}{c}19272 / 103 / \\
1179\end{array}$ & $\begin{array}{c}22331 / 66 / \\
1180\end{array}$ \\
\hline $\begin{array}{l}\text { Goodness-of-fit on } \\
F^{2}\end{array}$ & 0.904 & 0.884 & 1.061 & 0.918 \\
\hline$R_{1}, w R_{2}(I>2 \sigma(I))$ & $\begin{array}{l}R_{1}=0.0470, \\
W R_{2}=0.1244\end{array}$ & $\begin{array}{l}R_{1}=0.0507, \\
w R_{2}=0.1098\end{array}$ & $\begin{array}{l}R_{1}=0.0516, \\
w R_{2}=0.1310\end{array}$ & $\begin{array}{l}R_{1}=0.0447, \\
w R_{2}=0.1067\end{array}$ \\
\hline$R_{1}, w R_{2}$ (all data) & $\begin{array}{l}R_{1}=0.0604, \\
W R_{2}=0.1344\end{array}$ & $\begin{array}{l}R_{1}=0.0768, \\
w R_{2}=0.1208\end{array}$ & $\begin{array}{l}R_{1}=0.0616, \\
W R_{2}=0.1387\end{array}$ & $\begin{array}{c}R_{1}=0.0615, \\
w R_{2}=0.1154\end{array}$ \\
\hline $\begin{array}{l}\text { Largest diff. peak } \\
\text { and hole / e } \AA^{-3}\end{array}$ & $2.295 /-1.031$ & $1.937 /-1.808$ & $1.991 /-1.198$ & $1.957 /-1.268$ \\
\hline
\end{tabular}




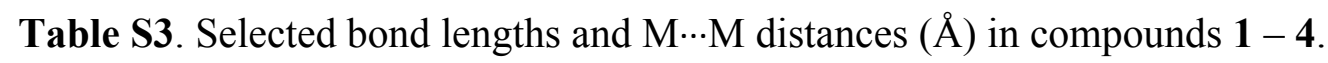

\begin{tabular}{|c|c|c|c|c|}
\hline \multirow{2}{*}{ Bond type } & \multicolumn{4}{|c|}{ Bond length $(\mathbf{M}-\mathbf{L})$ / Shortest distance $(\mathbf{M} \cdots \mathbf{M})$ / $\AA$} \\
\hline & 1 & 2 & 3 & 4 \\
\hline $\mathrm{Zr}-\mu_{3}-\mathrm{O}$ & $2.033(5)-2.364(5)$ & $2.048(2)-2.104(2)$ & $2.049(2)-2.108(2)$ & $2.051(4)-2.106(4)$ \\
\hline $\mathrm{Zr}-\mu_{4}-\mathrm{O}$ & $2.039(5)-2.183(5)$ & $2.117(2)-2.328(2)$ & $2.119(2)-2.324(2)$ & $2.110(4)-2.321(4)$ \\
\hline $\mathrm{Zr}-\mathrm{O}_{\mathrm{ib}}$ & $2.117(5)-2.168(5)$ & $2.103(2)-2.219(2)$ & $2.097(2)-2.212(2)$ & $2.104(4)-2.204(4)$ \\
\hline $\mathrm{Zr}-\mathrm{O}_{\text {bda }}$ & $2.112(5)-2.152(5)$ & $2.113(2)-2.161(2)$ & $2.111(2)-2.158(2)$ & $2.109(4)-2.170(4)$ \\
\hline $\mathrm{Ln}-\mu_{4}-\mathrm{O}$ & $2.537(5)$ & $2.541(2)$ & $2.522(2)$ & $2.504(4)$ \\
\hline $\mathrm{Ln}-\mathrm{O}_{\mathrm{NO} 3}$ & $2.560(6)-2.645(5)$ & $2.530(2)-2.631(2)$ & $2.504(2)-2.627(3)$ & $2.489(4)-2.614(4)$ \\
\hline $\mathrm{Ln}-\mathrm{O}_{\mathrm{ib} 1}$ & $2.460(6)-2.510(6)$ & $2.442(2)-2.452(2)$ & $2.422(3)-2.434(2)$ & $2.419(4)-2.421(4)$ \\
\hline $\mathrm{Ln}-\mathrm{O}_{\mathrm{ib} 2}$ & $2.629(6)-2.647(6)$ & $2.620(3)-2.629(3)$ & $2.595(3)-2.616(3)$ & $2.577(5)-2.600(4)$ \\
\hline $\mathrm{Ln}-\mathrm{O}_{\mathrm{ib} 3}$ & $2.735(5)-2.759(5)$ & $2.752(2)-2.777(2)$ & $2.751(2)-2.765(3)$ & $2.748(4)-2.761(4)$ \\
\hline $\mathrm{Fe}-\mu_{4}-\mathrm{O}$ & $1.980(5)$ & $1.975(2)$ & $1.972(2)$ & $1.980(4)$ \\
\hline $\mathrm{Fe}-\mathrm{N}$ & $2.232(7)$ & $2.199(3)$ & $2.200(3)$ & $2.201(4)$ \\
\hline $\mathrm{Fe}-\mathrm{O}_{\mathrm{bda}}$ & $1.994(6)-2.012(6)$ & $2.009(2)-2.028(2)$ & $2.007(2)-2.025(2)$ & $2.014(4)-2.033(4)$ \\
\hline $\mathrm{Fe}-\mathrm{O}_{\mathrm{ib}}$ & $1.976(6)-1.993(6)$ & $1.984(2)-2.008(2)$ & $1.988(2)-2.003(2)$ & $1.991(4)-2.009(4)$ \\
\hline $\mathrm{Zr} \cdots \mathrm{Zr}$ & $3.285(1)-3.461(1)$ & $3.313(4)-3.469(4)$ & $3.311(5)-3.466(5)$ & $3.315(1)-3.471(1)$ \\
\hline $\mathrm{Zr} \cdots \mathrm{Fe}$ & $3.285(1)-3.329(1)$ & $3.3080(5)$ & $3.3054(7)$ & $3.3156(11)$ \\
\hline $\mathrm{Zr} \cdots \operatorname{Ln}$ & 3.916 & 3.928 & 3.867 & 3.851 \\
\hline
\end{tabular}


Table S4. Selected bond lengths and $\mathrm{M} \cdots \mathrm{M}$ distances $(\AA)$ in compounds $5-\mathbf{8}$.

\begin{tabular}{|c|c|c|c|c|}
\hline \multirow{2}{*}{ Bond type } & \multicolumn{4}{|c|}{ 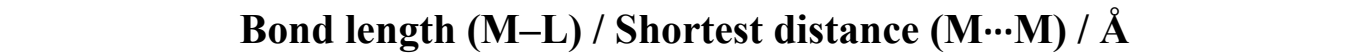 } \\
\hline & 5 & 6 & 7 & 8 \\
\hline $\mathrm{Zr}-\mu_{3}-\mathrm{O}$ & $2.030(4)-2.138(4)$ & $2.022(4)-2.131(4)$ & $2.030(4)-2.139(4)$ & $2.041(4)-2.138(3)$ \\
\hline $\mathrm{Zr}-\mu_{4}-\mathrm{O}$ & $2.080(3)-2.364(4)$ & $2.087(4)-2.351(4)$ & $2.068(3)-2.366(4)$ & $2.072(3)-2.359(3)$ \\
\hline $\mathrm{Zr}-\mathrm{O}_{\mathrm{ib}}$ & $2.111(4)-2.208(3)$ & $2.103(5)-2.204(4)$ & $2.082(5)-2.198(4)$ & $2.079(4)-2.207(3)$ \\
\hline $\mathrm{Zr}-\mathrm{O}_{\mathrm{bda}}$ & $2.107(4)-2.160(3)$ & $2.091(4)-2.165(4)$ & $2.102(4)-2.169(4)$ & $2.102(3)-2.158(3)$ \\
\hline $\mathrm{Ln}-\mu_{4}-\mathrm{O}$ & $2.551(3)-2.562(3)$ & $2.528(4)-2.549(4)$ & $2.511(3)-2.526(3)$ & $2.509(3)-2.519(3)$ \\
\hline $\mathrm{Ln}-\mathrm{O}_{\mathrm{NO} 3}$ & $2.582(4)-2.656(4)$ & $2.559(5)-2.645(4)$ & $2.541(4)-2.631(4)$ & $2.523(4)-2.613(3)$ \\
\hline $\mathrm{Ln}-\mathrm{O}_{\mathrm{ib} 1}$ & $2.421(4)-2.512(4)$ & $2.392(4)-2.479(5)$ & $2.368(4)-2.456(4)$ & $2.364(3)-2.575(4)$ \\
\hline $\mathrm{Ln}-\mathrm{O}_{\mathrm{ib} 2}$ & $2.640(4)-2.704(4)$ & $2.618(4)-2.713(5)$ & $2.579(5)-2.693(4)$ & $2.424(4)-2.689(4)$ \\
\hline $\mathrm{Ln}-\mathrm{O}_{\mathrm{ib} 3}$ & $2.752(3)-2.770(3)$ & $2.740(4)-2.773(4)$ & $2.745(4)-2.747(4)$ & $2.728(3)-2.754(3)$ \\
\hline $\mathrm{Ln} \cdots \mathrm{O}_{\mathrm{ib} 4}$ & $2.729(4)$ & $2.716(5)$ & $2.654(4)$ & $2.640(4)$ \\
\hline $\mathrm{Ln} \cdots \mathrm{O}_{\mathrm{ib} 5}$ & $2.825(4)$ & $2.857(5)$ & $3.015(4)$ & $3.991(4)$ \\
\hline $\mathrm{Fe}-\mu_{4}-\mathrm{O}$ & $1.959(3)-1.969(3)$ & $1.960(4)-1.968(4)$ & $1.953(4)-1.957(3)$ & $1.961(3)-1.965(3)$ \\
\hline $\mathrm{Fe}-\mathrm{N}$ & $2.178(5)-2.184(4)$ & $2.168(5)-2.179(5)$ & $2.173(5)-2.175(5)$ & $2.172(4)-2.178(4)$ \\
\hline $\mathrm{Fe}-\mathrm{O}_{\mathrm{bda}}$ & $2.005(3)-2.033(4)$ & $1.998(5)-2.036(4)$ & $1.997(4)-2.026(4)$ & $2.007(4)-2.030(4)$ \\
\hline $\mathrm{Fe}-\mathrm{O}_{\mathrm{ib}}$ & $1.980(4)-2.020(4)$ & $1.989(5)-2.014(5)$ & $1.978(4)-2.015(4)$ & $1.986(4)-2.019(4)$ \\
\hline $\mathrm{Zr} \cdots \mathrm{Zr}$ & $3.308(7)-3.484(8)$ & $3.311(1)-3.486(1)$ & $3.315(1)-3.502(1)$ & $3.315(1)-3.515(2)$ \\
\hline $\mathrm{Zr} \cdots \mathrm{Fe}$ & $3.291(1)-3.322(1)$ & $3.292(1)-3.323(1)$ & $3.287(1)-3.327(1)$ & $3.290(1)-3.295(1)$ \\
\hline $\mathrm{Zr} \cdots \operatorname{Ln}$ & $3.941(7)-3.944(6)$ & $3.887(2)-3.903(2)$ & $3.863(1)-3.886(1)$ & $3.870(3)-3.871(1)$ \\
\hline
\end{tabular}




\section{Bond valence sum calculations for compounds $\mathbf{1}$ - $\mathbf{8}$}

Bond valence sum calculations ${ }^{[1,2]}$ for $\mathbf{1}-\mathbf{4}$ (Table S5) and $\mathbf{5}-\mathbf{8}$ (Table S6) are consistent with the + IV oxidation states for all $\mathrm{Zr}$ and + III oxidation states for all $\mathrm{Ln}$ and Fe centers in $\mathbf{1}-\mathbf{8}$. The calculated BVS values for oxygen atoms of the $\left\{\mathrm{Zr}_{6} \mathrm{O}_{8}\right\}$ core in these complexes do not suggest any protonation.

Table S5. Bond valence sum values for metal centers and $\mathrm{O}$ atoms of the central $\left\{\mathrm{Zr}_{6} \mathrm{O}_{8}\right\}$ core in $\mathbf{1}-\mathbf{4}$ (space group $P 2_{1} / n$ ).

\begin{tabular}{|l|c|c|c|c|}
\hline \multirow{2}{*}{ Atom } & \multicolumn{4}{|c|}{ BVS values } \\
\hline Zr1 & $\mathbf{1}$ & $\mathbf{2}$ & $\mathbf{3}$ & $\mathbf{4}$ \\
\hline $\mathrm{Zr} 2$ & 4.14 & 4.07 & 4.09 & 4.07 \\
\hline $\mathrm{Zr3}$ & 4.14 & 4.07 & 4.07 & 4.03 \\
\hline $\mathrm{Ln} 1$ & 4.10 & 4.05 & 4.06 & 4.06 \\
\hline Fe1 & 3.28 & 3.27 & 3.30 & 3.22 \\
\hline O1 & 3.09 & 3.05 & 3.06 & 3.01 \\
\hline O2 & -2.04 & -1.92 & -1.93 & -1.91 \\
\hline O3 & -1.99 & -1.97 & -1.97 & -2.03 \\
\hline O4 & -2.09 & -2.04 & -2.04 & -2.08 \\
\hline
\end{tabular}


Table S6. Bond valence sum values for metal centers and $\mathrm{O}$ atoms of the central $\left\{\mathrm{Zr}_{6} \mathrm{O}_{8}\right\}$ core in 5 - 8 (space group $P-1$ ).

\begin{tabular}{|l|c|c|c|c|}
\hline \multirow{2}{*}{ Atom } & \multicolumn{4}{|c|}{ BVS value for the compound } \\
\hline Zr1 & $\mathbf{5}$ & $\mathbf{6}$ & $\mathbf{7}$ & $\mathbf{8}$ \\
\hline Zr2 & 4.10 & 4.06 & 4.13 & 4.09 \\
\hline Zr3 & 4.09 & 4.09 & 4.07 & 4.09 \\
\hline Zr4 & 4.05 & 4.12 & 4.08 & 4.06 \\
\hline Zr5 & 4.10 & 4.11 & 4.07 & 4.05 \\
\hline Zr6 & 4.08 & 4.11 & 4.11 & 4.12 \\
\hline Ln1 & 4.07 & 4.09 & 4.10 & 4.06 \\
\hline Ln2 & 3.22 & 3.18 & 3.22 & 3.15 \\
\hline Fe1 & 3.21 & 3.24 & 3.24 & 3.19 \\
\hline Fe2 & 3.07 & 3.00 & 3.09 & 3.04 \\
\hline O1 & 3.04 & 3.09 & 3.11 & 3.07 \\
\hline O2 & -1.94 & -1.94 & -1.95 & -1.93 \\
\hline O3 & -2.00 & -2.10 & -2.00 & -2.00 \\
\hline O4 & -2.01 & -2.01 & -2.01 & -1.99 \\
\hline O5 & -2.14 & -2.13 & -2.14 & -2.12 \\
\hline O6 & -1.96 & -1.95 & -1.93 & -1.89 \\
\hline O7 & -2.00 & -2.02 & -2.10 & -2.00 \\
\hline O8 & -2.08 & -2.09 & -2.09 & -2.08 \\
\hline
\end{tabular}




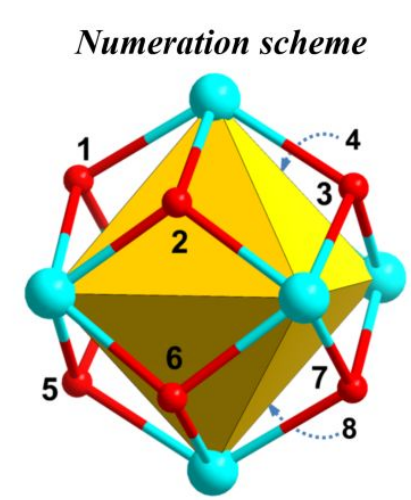

$\left\{\mathrm{Zr}_{6} \mathrm{O}_{8}\right\}$ core

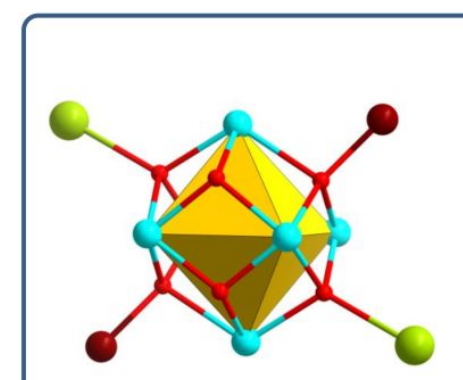

$\left\{\left(1,7-\mathrm{Ln}_{2}{ }^{\mathrm{III}}\right)\left(3,5-\mathrm{Fe}^{\mathrm{III}}{ }_{2}\right)\left(\mathrm{Zr}_{6} \mathrm{O}_{8}\right)\right\}$

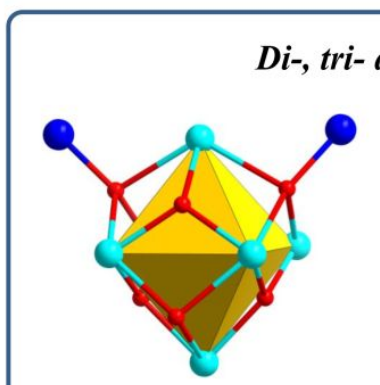

$\left\{\left(1,3-\mathrm{M}^{\mathrm{III}}{ }_{2}\right)\left(\mathrm{Zr}_{6} \mathrm{O}_{6}(\mathrm{OH})_{2}\right\}\right.$ $(\mathrm{M}=\mathrm{Fe}, \mathrm{Mn})$

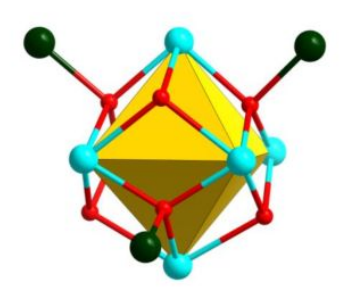

$\left\{\left(1,3,6-\mathrm{Ni}^{\mathrm{II}}\right)\left(\mathrm{Zr}_{6} \mathrm{O}_{4}(\mathrm{OH})_{4}\right\}\right.$

Tetra-metalated $\left\{\mathrm{Zr}_{6} \mathrm{O}_{8}\right\}$ and $\left\{\mathrm{PbZr}_{5} \mathrm{O}_{8}\right\}$ cores

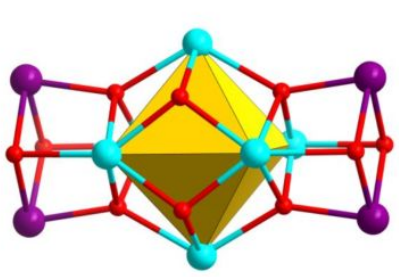

$\left\{\left(1,3,5,7-\mathrm{M}_{4}{ }_{4}\right)\left(\mathrm{Zr}_{6} \mathrm{O}_{8}\right)\right\}$

$(\mathrm{M}=\mathrm{Co}, \mathrm{Ni})$

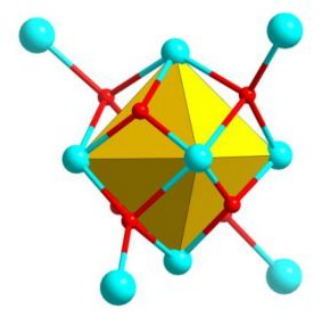

$\left\{\left(1,3,6,8-\mathrm{Zr}^{\mathrm{IV}}{ }_{4}\right)\left(\mathrm{Zr}_{6} \mathrm{O}_{8}\right)\right\}$

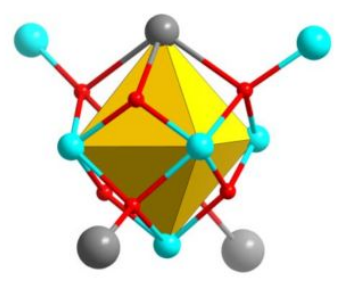

$\left\{\left(1,3-\mathrm{Zr}^{\mathrm{IV}}{ }_{2}\right)\left(6,8-\mathrm{Pb}_{2}{ }_{2}\right)-\right.$ $\left.\left(\operatorname{PbZr}_{5} \mathrm{O}_{8}\right)\right\}$

Figure S1. Proposed numeration scheme for extended $\left\{\mathrm{Zr}_{6} \mathrm{O}_{8}\right\}$ structures and comparison of the known metalated $\left\{\mathrm{Zr}_{6} \mathrm{O}_{8}\right\}$ cores, $\left\{\mathrm{M}_{x} \mathrm{Zr}^{\mathrm{IV}}{ }_{6} \mathrm{O}_{8} \mathrm{H}_{y}\right\}$ (see Table 1 in the main text). 


\section{IR spectra of compounds $\mathbf{1}-\mathbf{8}$}

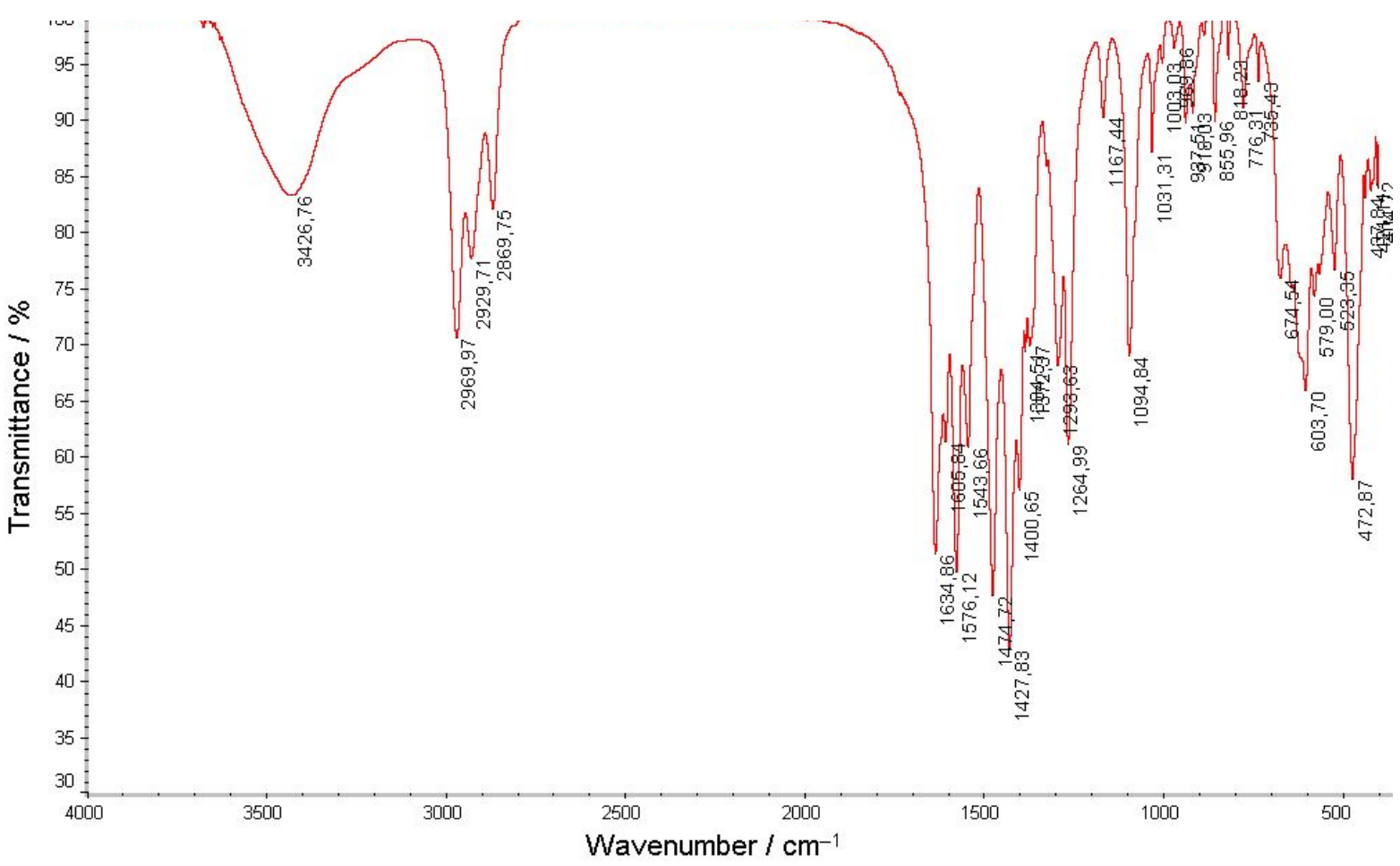

Figure S2. IR spectrum of compound 1 measured in the $4000-450 \mathrm{~cm}^{-1}$ region, using a $\mathrm{KBr}$ pellet.

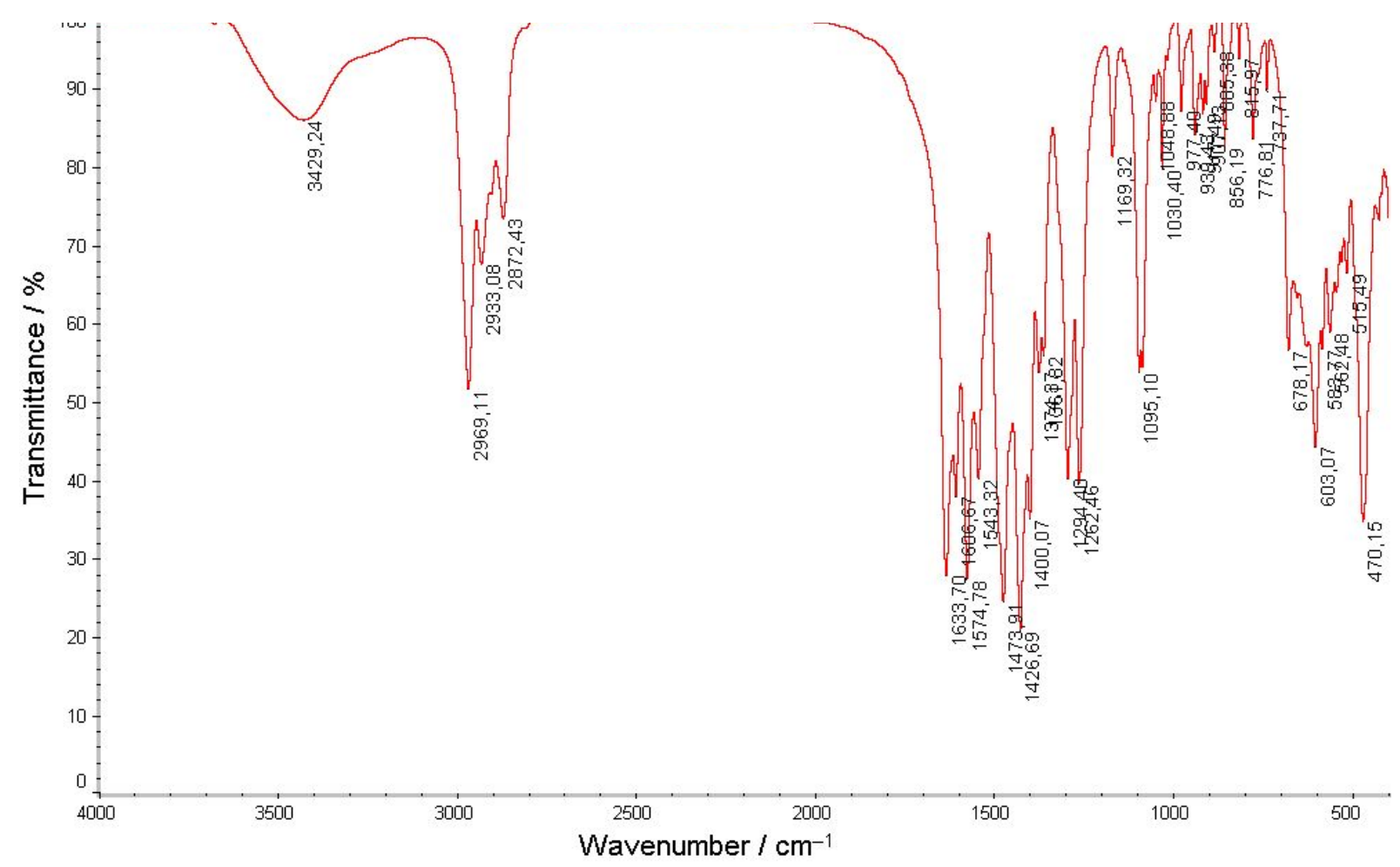

Figure S3. IR spectrum of compound 2 measured in the $4000-450 \mathrm{~cm}^{-1}$ region, using a $\mathrm{KBr}$ pellet. 


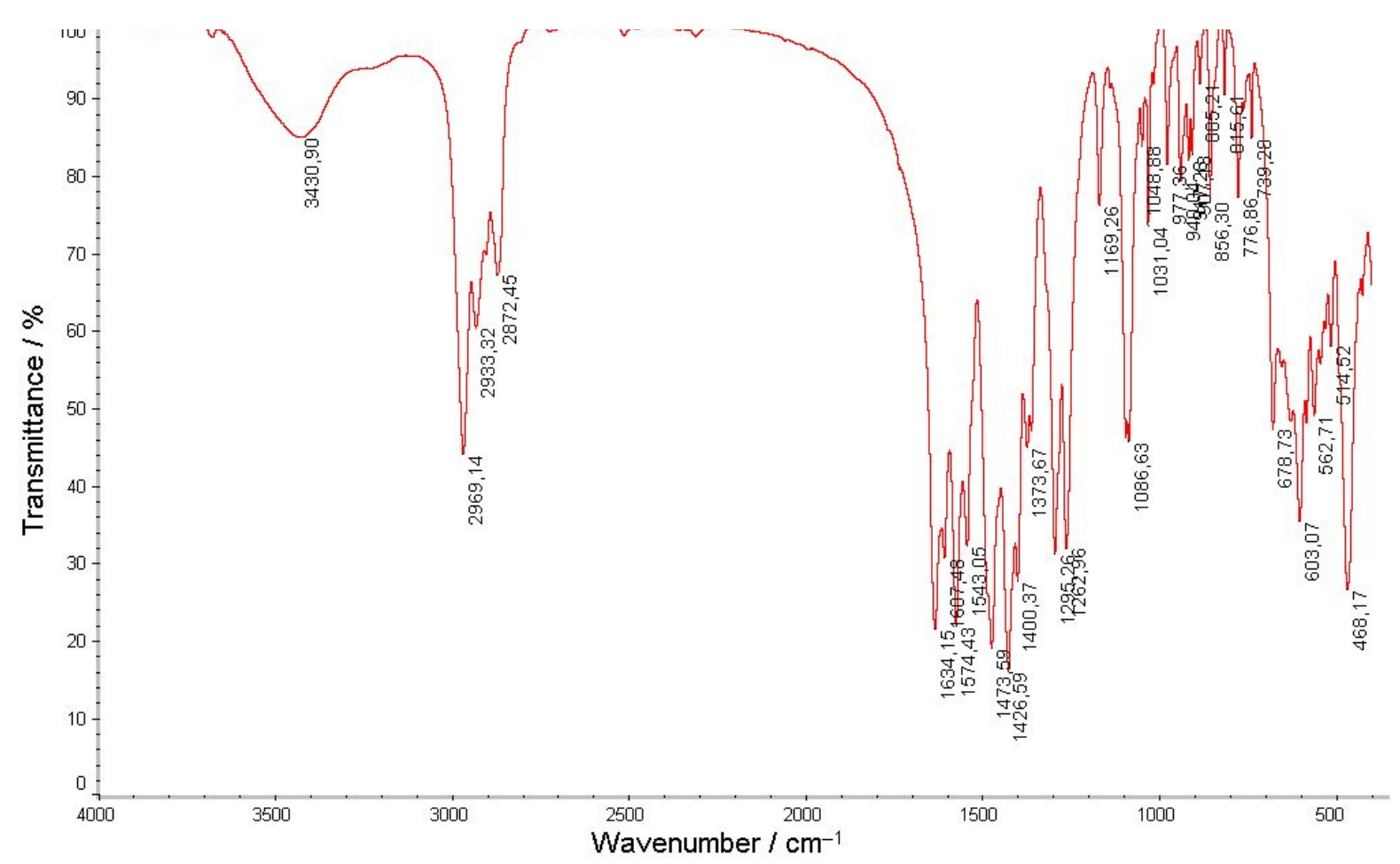

Figure S4. IR spectrum of compound 3 measured in the $4000-450 \mathrm{~cm}^{-1}$ region, using a $\mathrm{KBr}$ pellet.

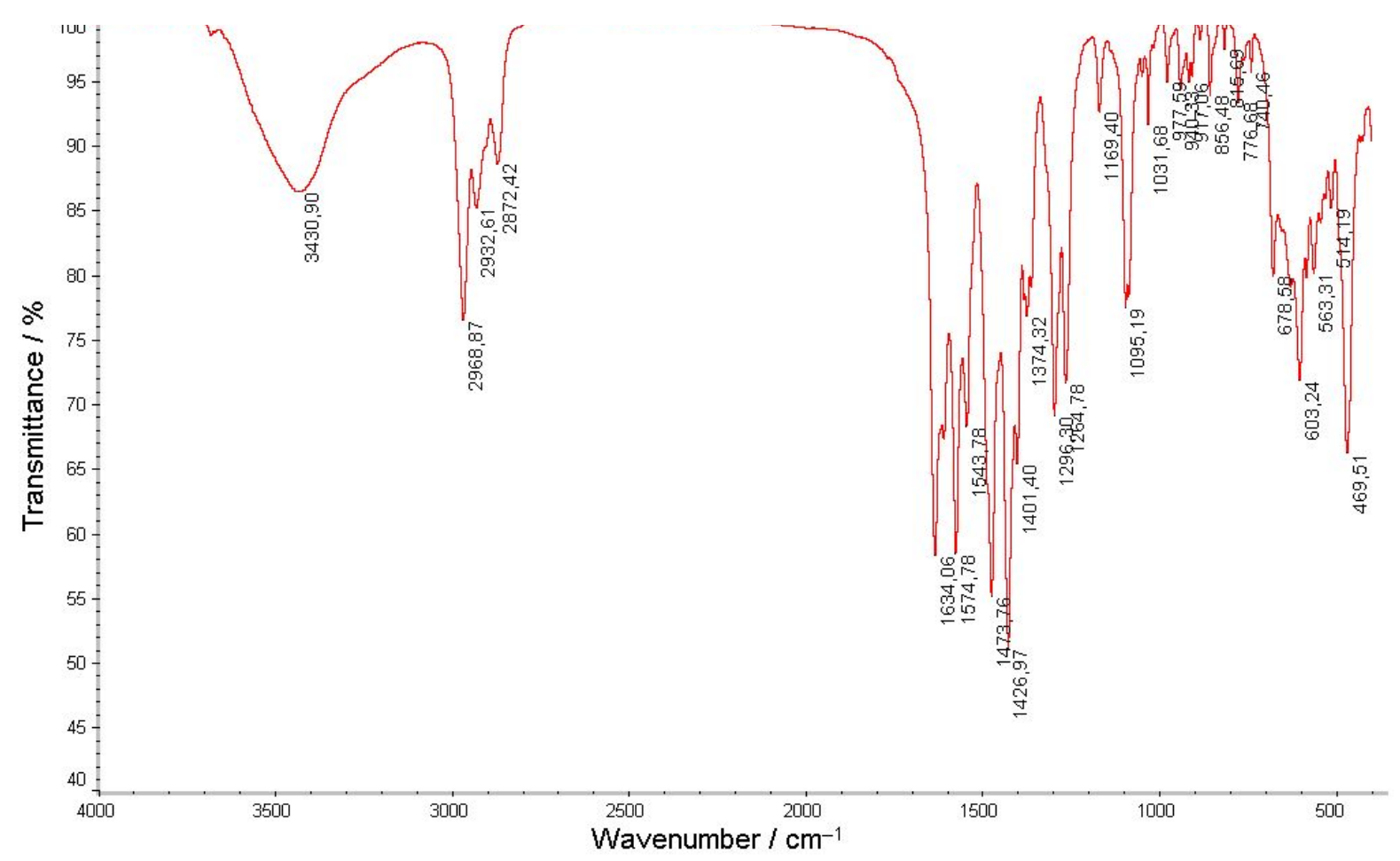

Figure S5. IR spectrum of compound 4 measured in the $4000-450 \mathrm{~cm}^{-1}$ region, using a $\mathrm{KBr}$ pellet. 


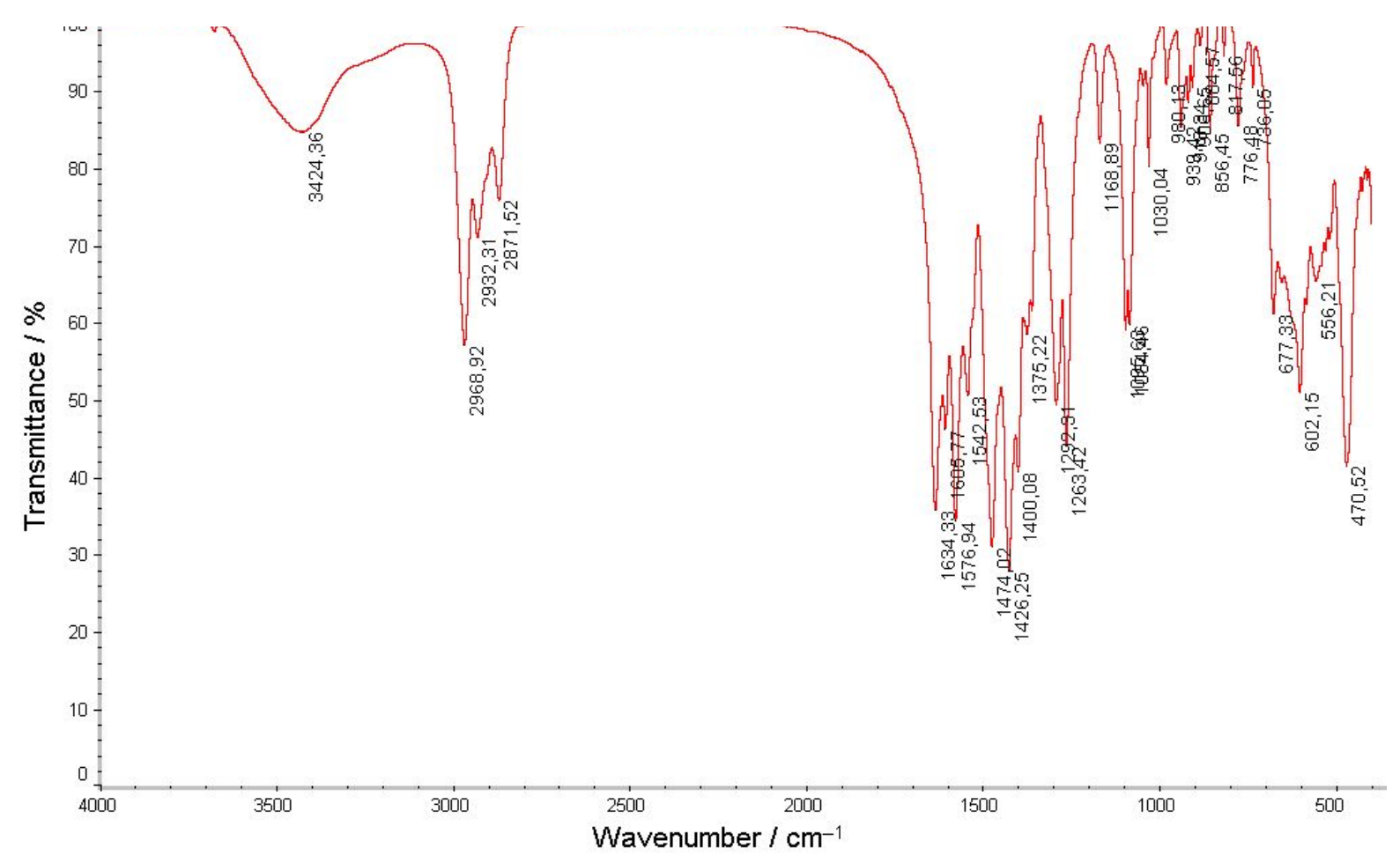

Figure S6. IR spectrum of compound 5 measured in the $4000-450 \mathrm{~cm}^{-1}$ region, using a $\mathrm{KBr}$ pellet.

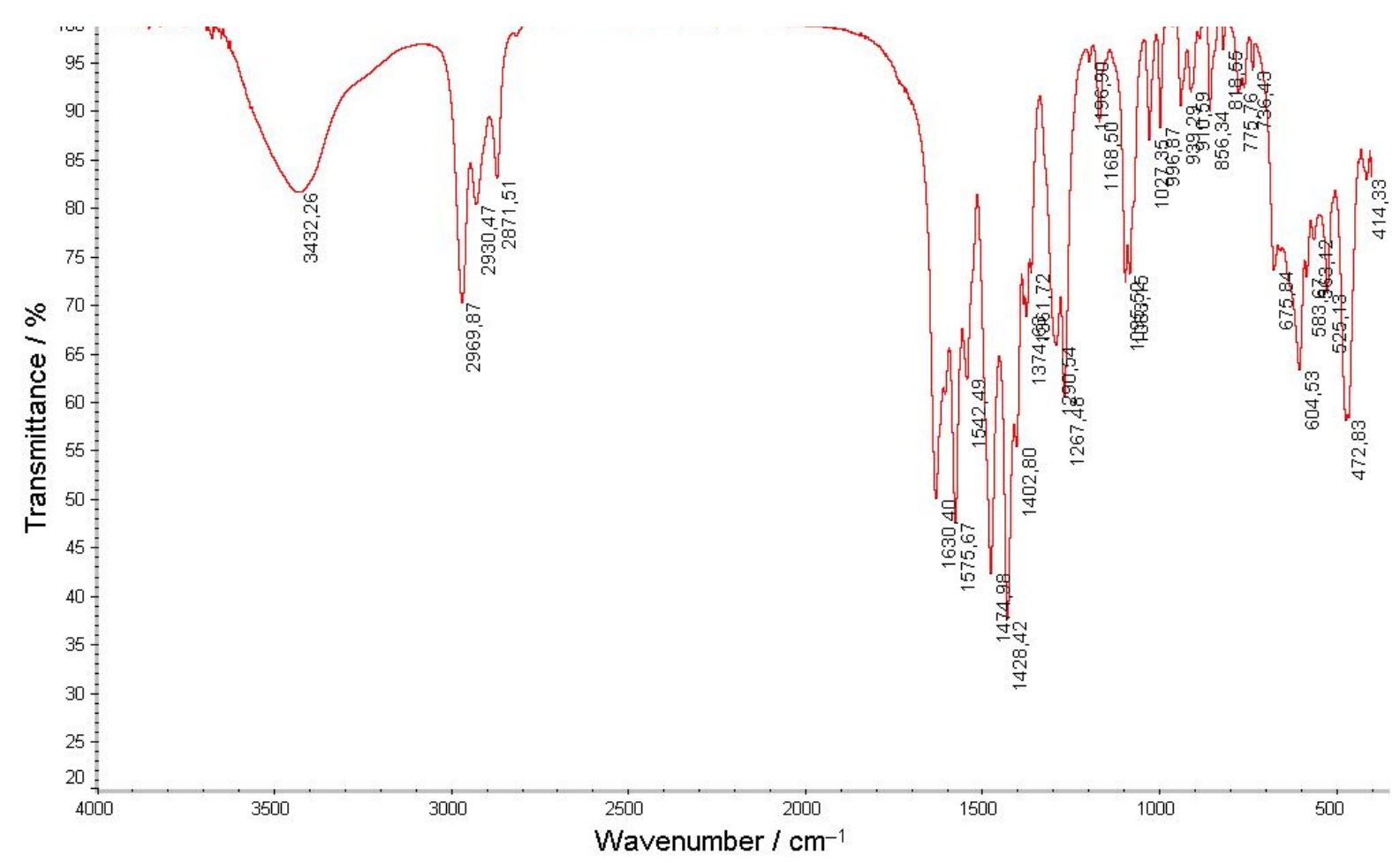

Figure S7. IR spectrum of compound 6 measured in the $4000-450 \mathrm{~cm}^{-1}$ region, using a $\mathrm{KBr}$ pellet. 


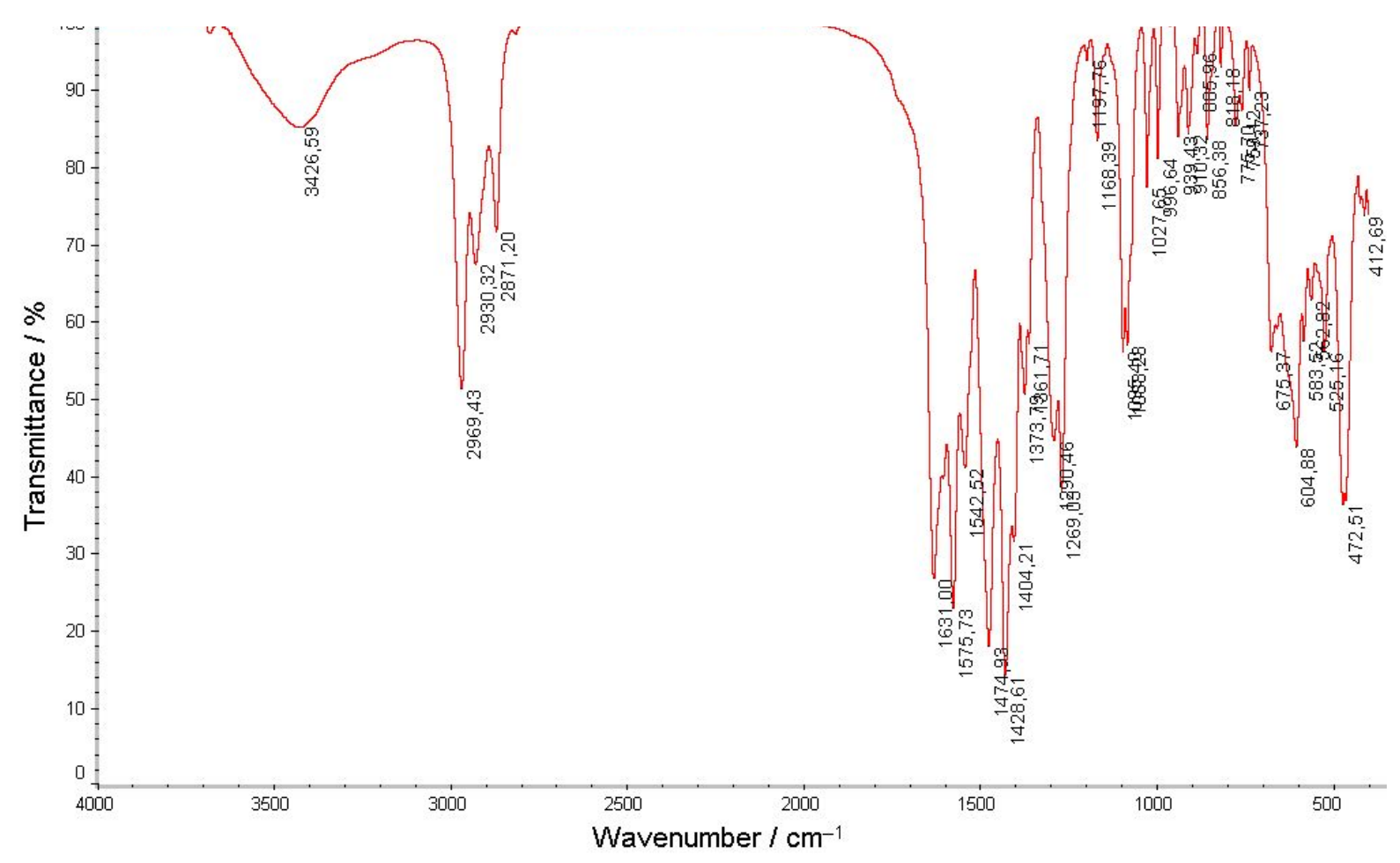

Figure S8. IR spectrum of compound 7 measured in the $4000-450 \mathrm{~cm}^{-1}$ region, using a $\mathrm{KBr}$ pellet.

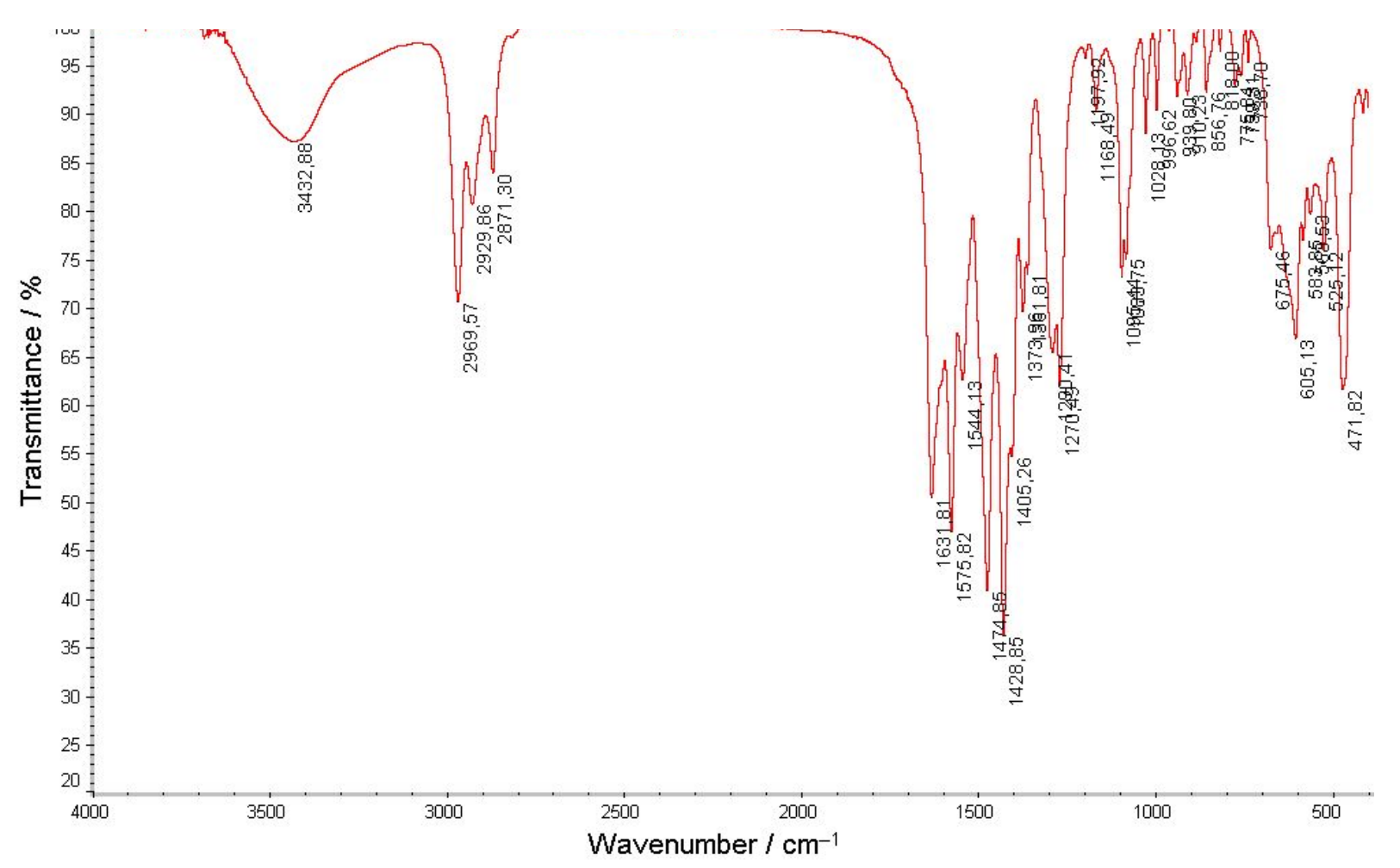

Figure S9. IR spectrum of compound 8 measured in the $4000-450 \mathrm{~cm}^{-1}$ region, using a $\mathrm{KBr}$ pellet. 
TGA/DTA curves of compounds $\mathbf{1}-\mathbf{8}$

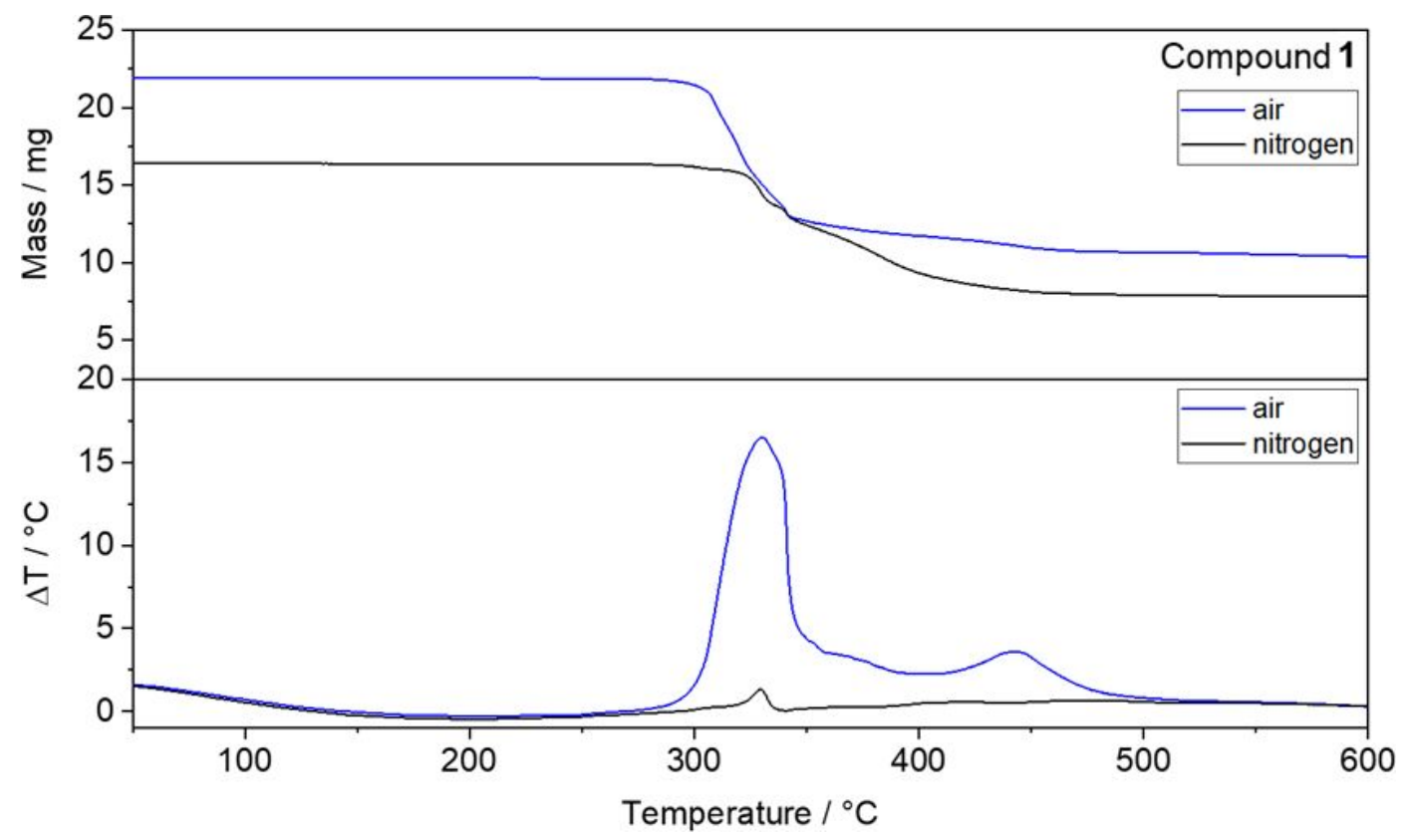

Figure S10. Top: TGA curves of compound 1 measured in dry air (blue) and under nitrogen atmosphere (black). Bottom: DTA curves of compound 1 measured in dry air (blue) and under nitrogen atmosphere (black).

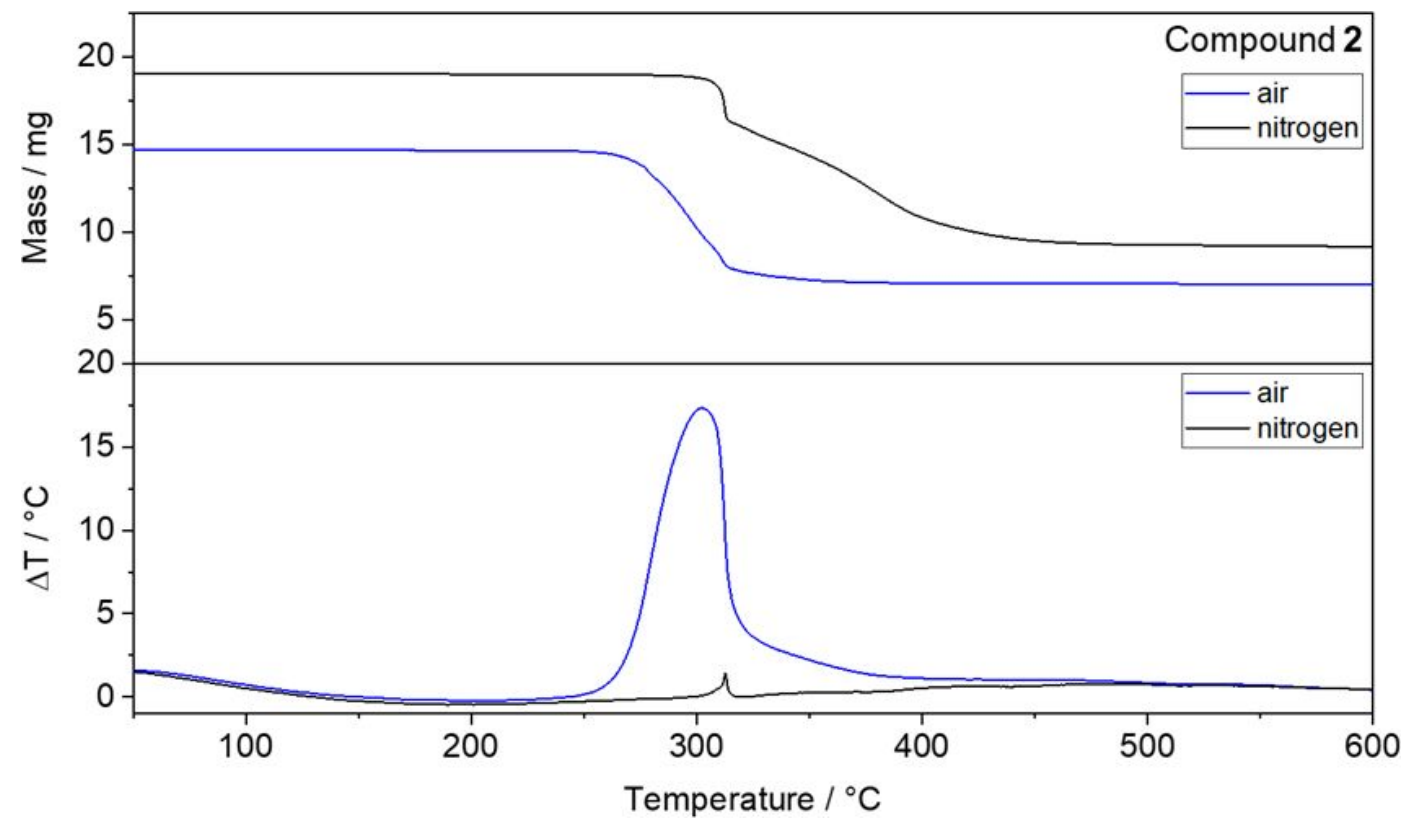

Figure S11. Top: TGA curves of compound 2 measured in dry air (blue) and under nitrogen atmosphere (black). Bottom: DTA curves of compound 2 measured in dry air (blue) and under nitrogen atmosphere (black). 


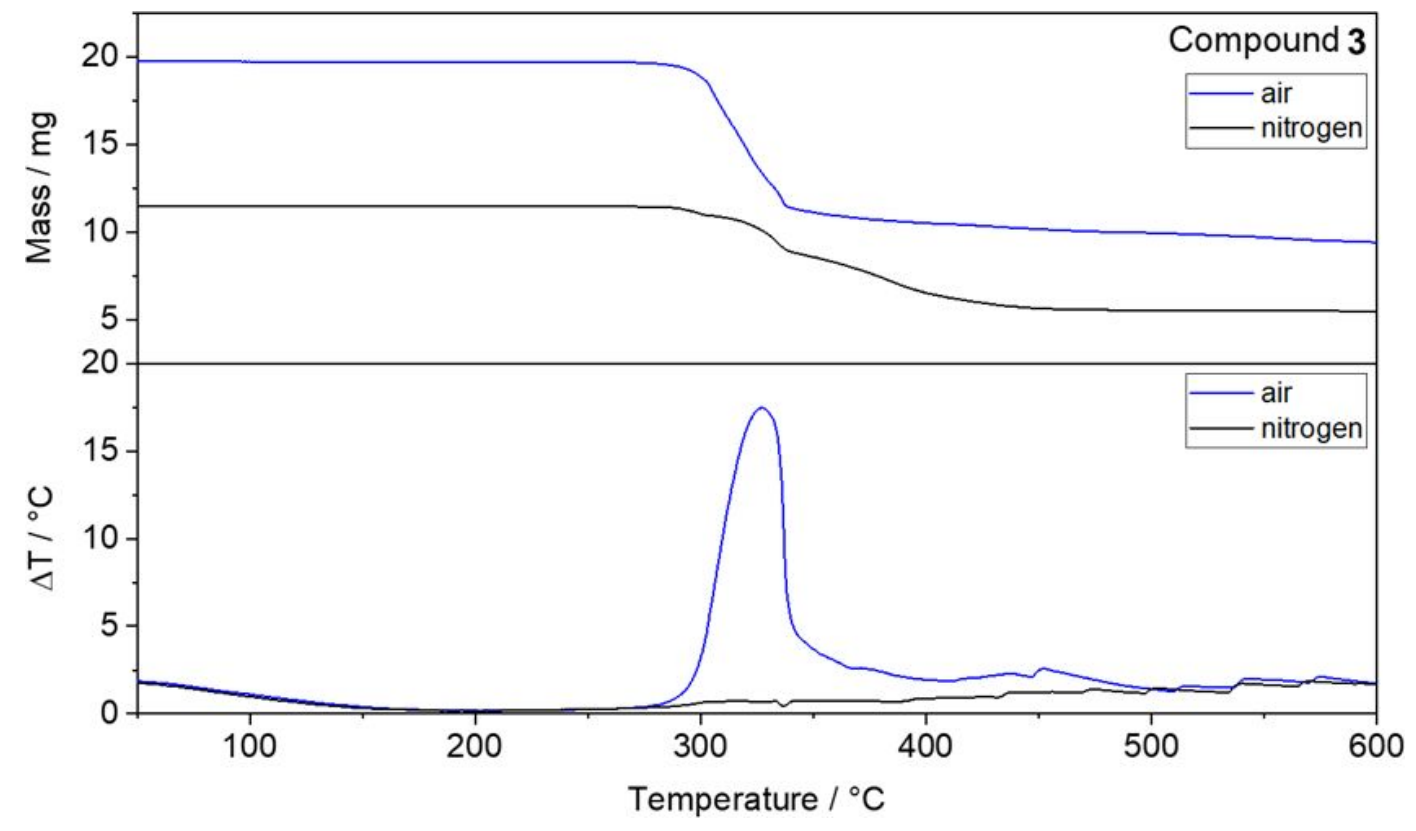

Figure S12. Top: TGA curves of compound 3 measured in dry air (blue) and under nitrogen atmosphere (black). Bottom: DTA curves of compound 3 measured in dry air (blue) and under nitrogen atmosphere (black).

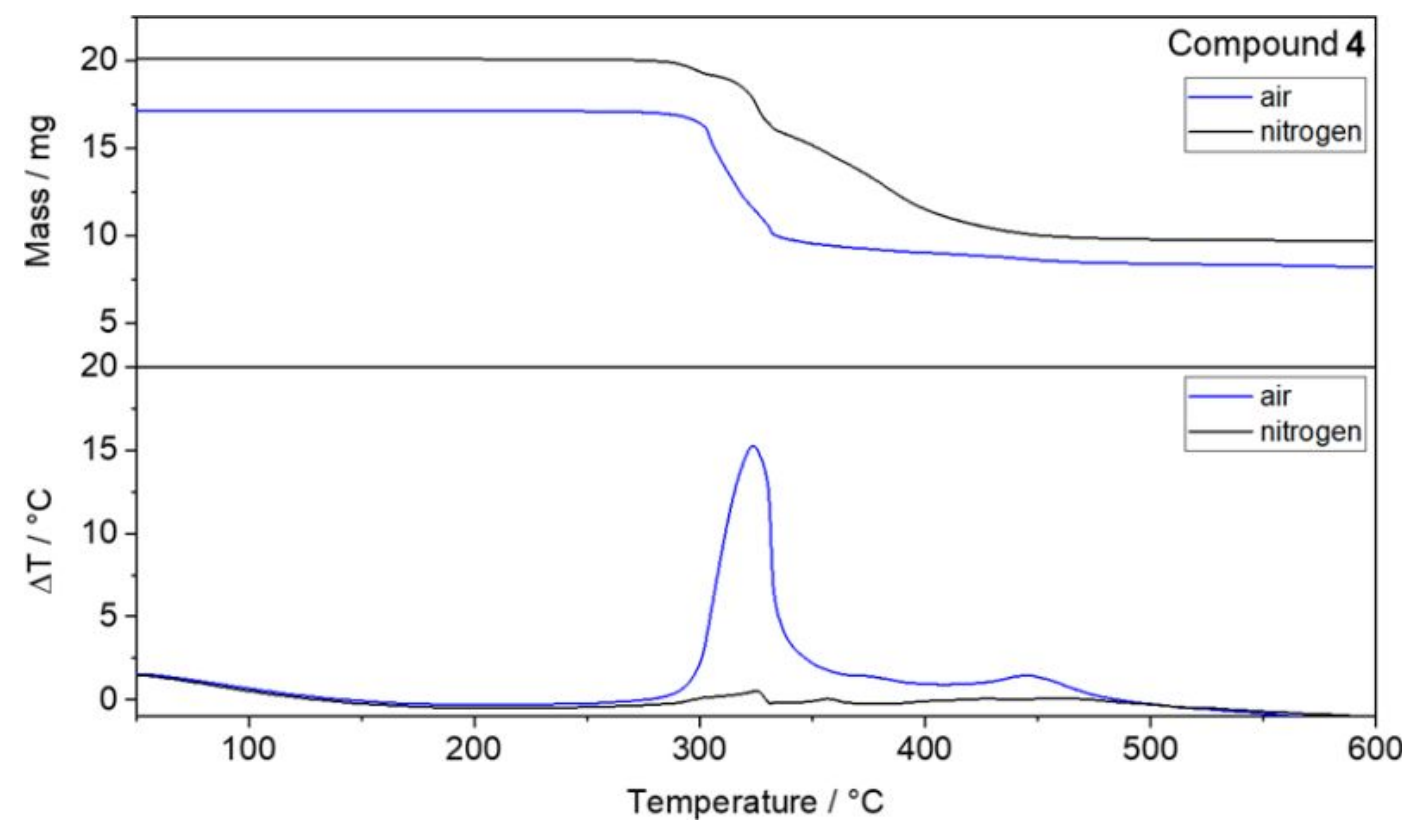

Figure S13. Top: TGA curves of compound $\mathbf{4}$ measured in dry air (blue) and under nitrogen atmosphere (black). Bottom: DTA curves of compound $\mathbf{4}$ measured in dry air (blue) and under nitrogen atmosphere (black). 


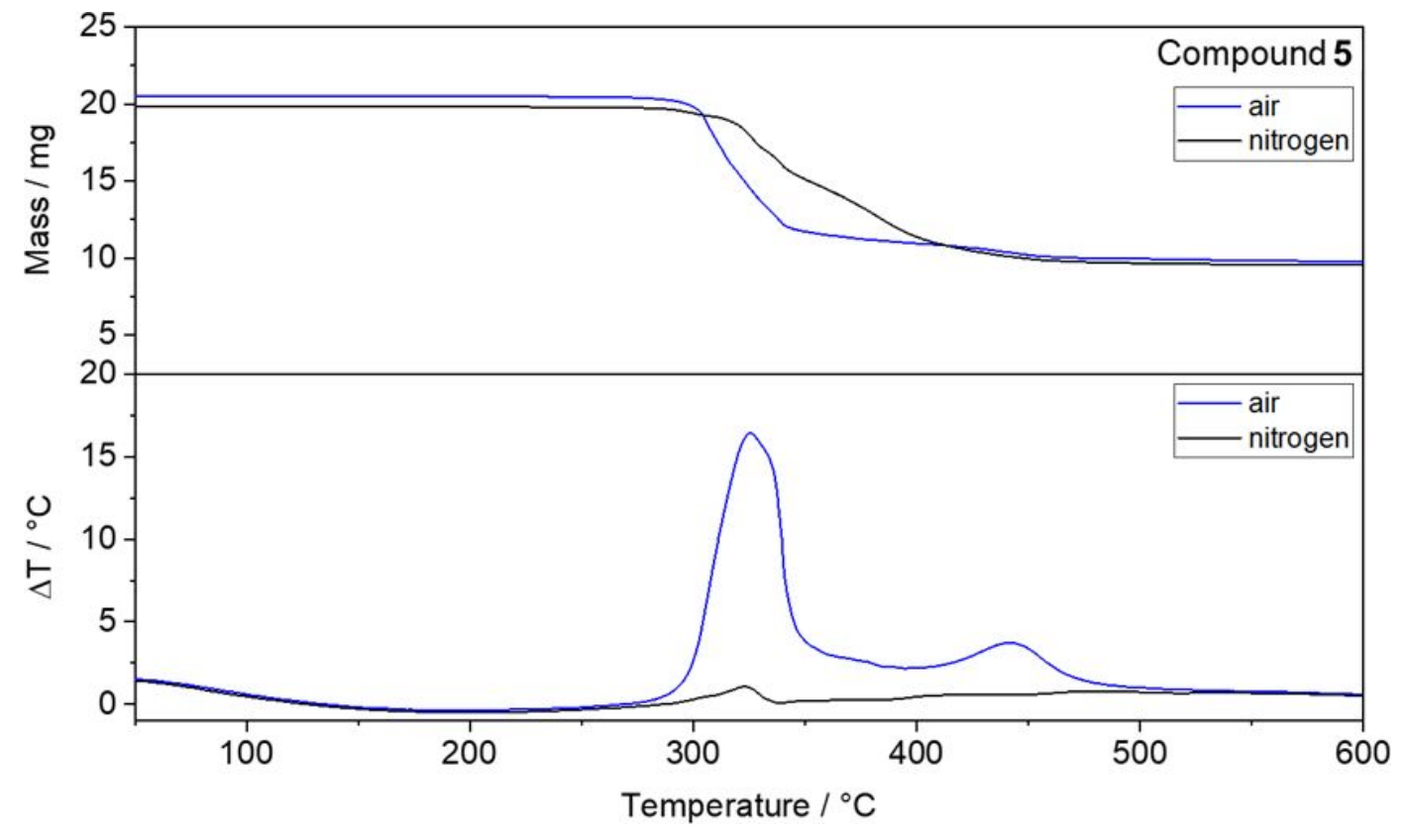

Figure S14. Top: TGA curves of compound 5 measured in dry air (blue) and under nitrogen atmosphere (black). Bottom: DTA curves of compound 5 measured in dry air (blue) and under nitrogen atmosphere (black).

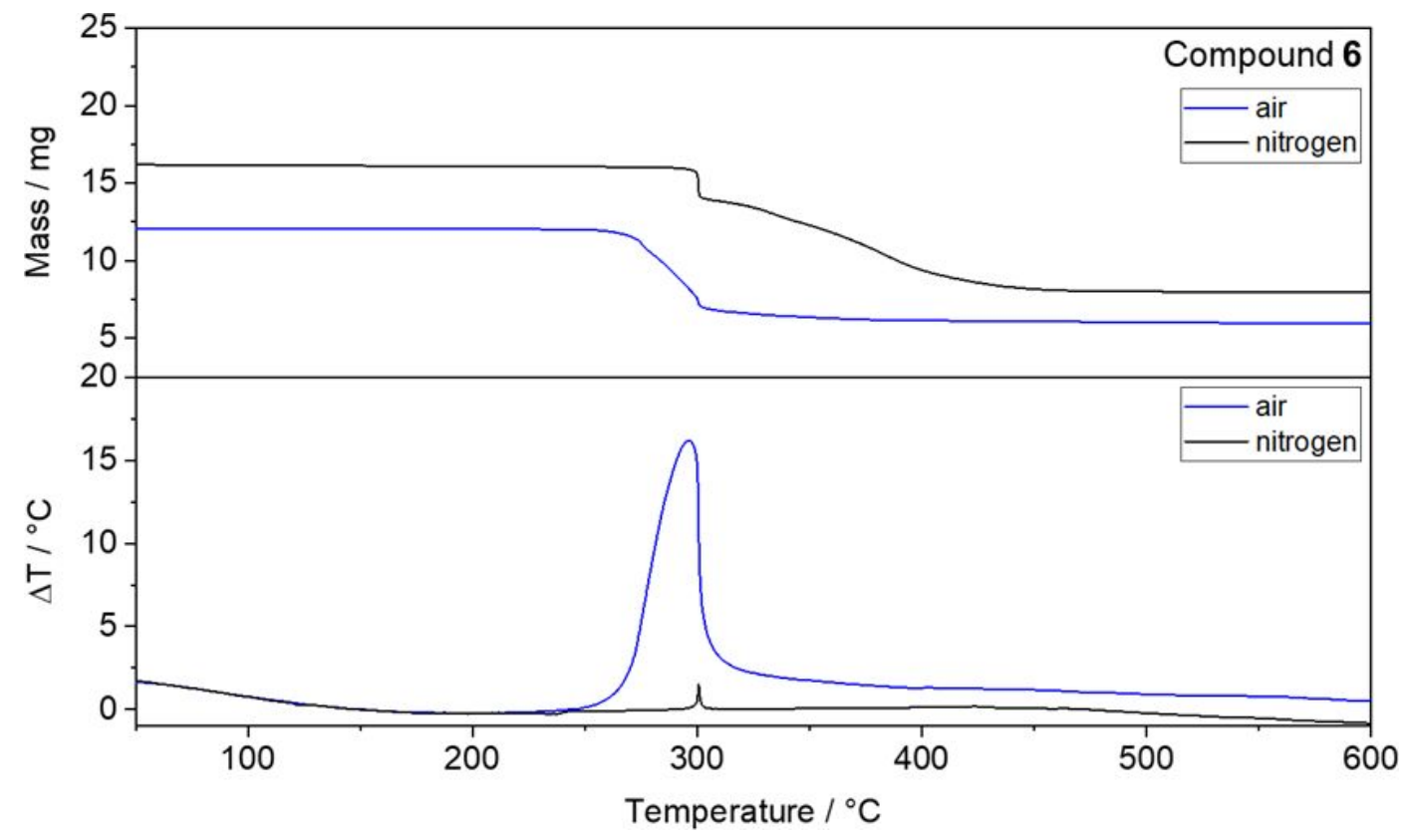

Figure S15. Top: TGA curves of compound $\mathbf{6}$ measured in dry air (blue) and under nitrogen atmosphere (black). Bottom: DTA curves of compound $\mathbf{6}$ measured in dry air (blue) and under nitrogen atmosphere (black). 


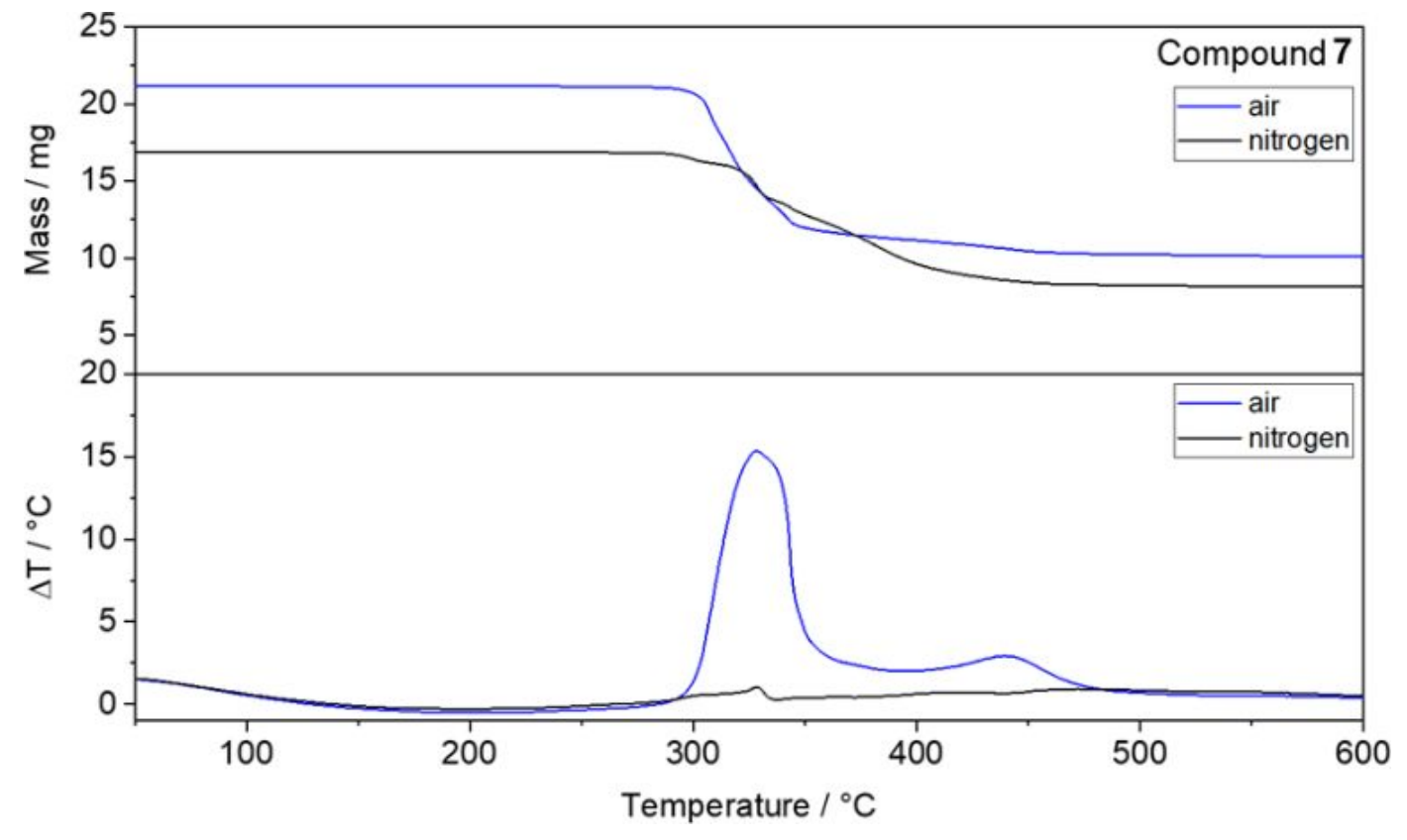

Figure S16. Top: TGA curves of compound 7 measured in dry air (blue) and under nitrogen atmosphere (black). Bottom: DTA curves of compound 7 measured in dry air (blue) and under nitrogen atmosphere (black).

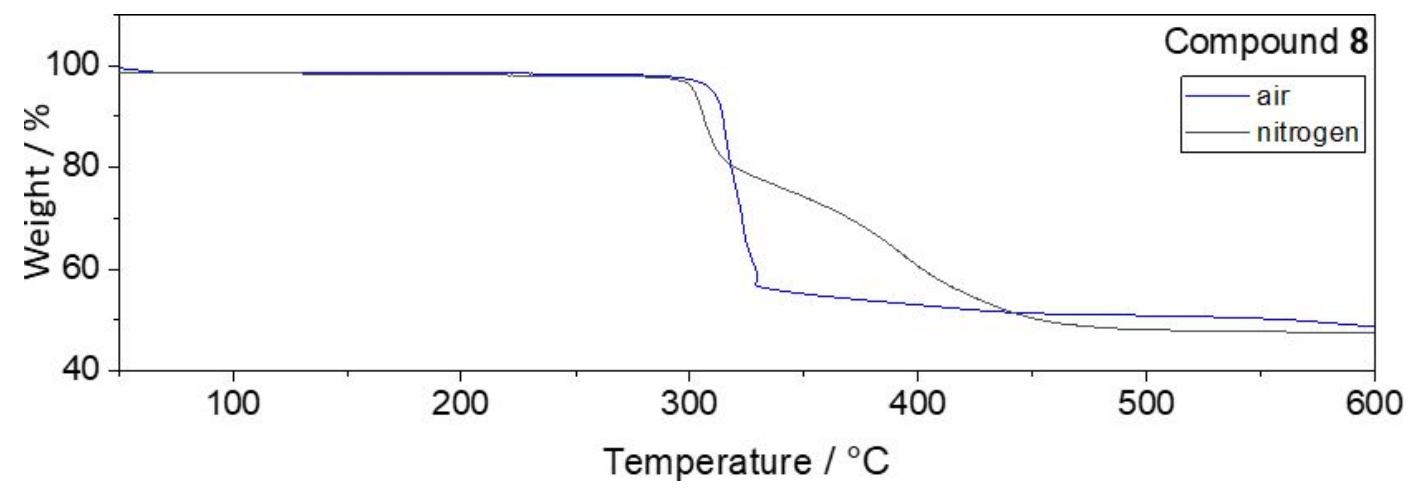

Figure S17. TGA curves of compound $\mathbf{8}$ measured in dry air (blue) and under nitrogen atmosphere (black). 
Table S7. Measured and calculated mass loss steps of $\mathbf{1}-\mathbf{8}$ in the temperature range 25$600{ }^{\circ} \mathrm{C}$ under a flow of dry air and nitrogen.

\begin{tabular}{|l|l|l|l|l|}
\hline compound & total $\Delta \boldsymbol{m}_{\text {meas. }} / \%$ & $\Delta \boldsymbol{m}_{\text {calc. }} / \%$ & deviation / \% & assignment \\
\hline \multicolumn{5}{|l|}{ in air } \\
\hline $\mathbf{1}$ & 52.5 & 55.1 & 2.6 & $-\mathrm{C}_{72} \mathrm{H}_{132} \mathrm{~N}_{4} \mathrm{O}_{28}$ \\
\hline $\mathbf{2}$ & 52.3 & 55.0 & 2.7 & $-\mathrm{C}_{72} \mathrm{H}_{132} \mathrm{~N}_{4} \mathrm{O}_{28}$ \\
\hline $\mathbf{3}$ & 52.4 & 55.0 & 2.6 & $-\mathrm{C}_{72} \mathrm{H}_{132} \mathrm{~N}_{4} \mathrm{O}_{28}$ \\
\hline $\mathbf{4}$ & 52.2 & 54.9 & 2.7 & $-\mathrm{C}_{72} \mathrm{H}_{132} \mathrm{~N}_{4} \mathrm{O}_{28}$ \\
\hline $\mathbf{5}$ & 52.4 & 53.6 & 1.2 & $-\mathrm{C}_{66} \mathrm{H}_{120} \mathrm{~N}_{4} \mathrm{O}_{28}$ \\
\hline $\mathbf{6}$ & 50.5 & 53.6 & 3.1 & $-\mathrm{C}_{66} \mathrm{H}_{120} \mathrm{~N}_{4} \mathrm{O}_{28}$ \\
\hline $\mathbf{7}$ & 52.4 & 53.6 & 1.2 & $-\mathrm{C}_{66} \mathrm{H}_{120} \mathrm{~N}_{4} \mathrm{O}_{28}$ \\
\hline $\mathbf{8}$ & 51.6 & 55.0 & 3.4 & $-\mathrm{C}_{68} \mathrm{H}_{123} \mathrm{~N}_{5} \mathrm{O}_{28}$ \\
\hline & & 53.6 & & \\
\hline $\mathbf{1}$ & 52.3 & 55.1 & 2.8 & $-\mathrm{C}_{72} \mathrm{H}_{132} \mathrm{~N}_{4} \mathrm{O}_{28}$ \\
\hline $\mathbf{2}$ & 51.9 & 55.0 & 3.1 & $-\mathrm{C}_{72} \mathrm{H}_{132} \mathrm{~N}_{4} \mathrm{O}_{28}$ \\
\hline $\mathbf{3}$ & 52.3 & 55.0 & 2.7 & $-\mathrm{C}_{72} \mathrm{H}_{132} \mathrm{~N}_{4} \mathrm{O}_{28}$ \\
\hline $\mathbf{4}$ & 52.0 & 54.9 & 2.9 & $-\mathrm{C}_{72} \mathrm{H}_{132} \mathrm{~N}_{4} \mathrm{O}_{28}$ \\
\hline $\mathbf{5}$ & 51.8 & 53.6 & $-\mathrm{C}_{66} \mathrm{H}_{120} \mathrm{~N}_{4} \mathrm{O}_{28}$ \\
\hline $\mathbf{6}$ & 51.1 & 53.6 & $-\mathrm{C}_{66} \mathrm{H}_{120} \mathrm{~N}_{4} \mathrm{O}_{28}$ \\
\hline $\mathbf{8}$ & 51.9 & 53.6 & $\mathrm{H}_{120} \mathrm{~N}_{4} \mathrm{O}_{28}$ \\
\hline
\end{tabular}




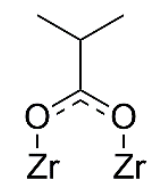

syn, syn- $-\eta^{1}: \eta^{1}: \mu_{2}$<smiles></smiles>

$$
\eta^{2}: \eta^{1}: \mu_{2}
$$<smiles>CCCOC(=O)C(C)C</smiles><smiles>[Z7]OC(=O)C(C)C</smiles>

syn, syn- $\eta^{1}: \eta^{1}: \mu_{2}$

Figure S18. Schematic representation of isobutyrate-metal coordination variations in 1-8, which also manifest in distinct carboxylate vibration modes in the IR spectra of the compounds.

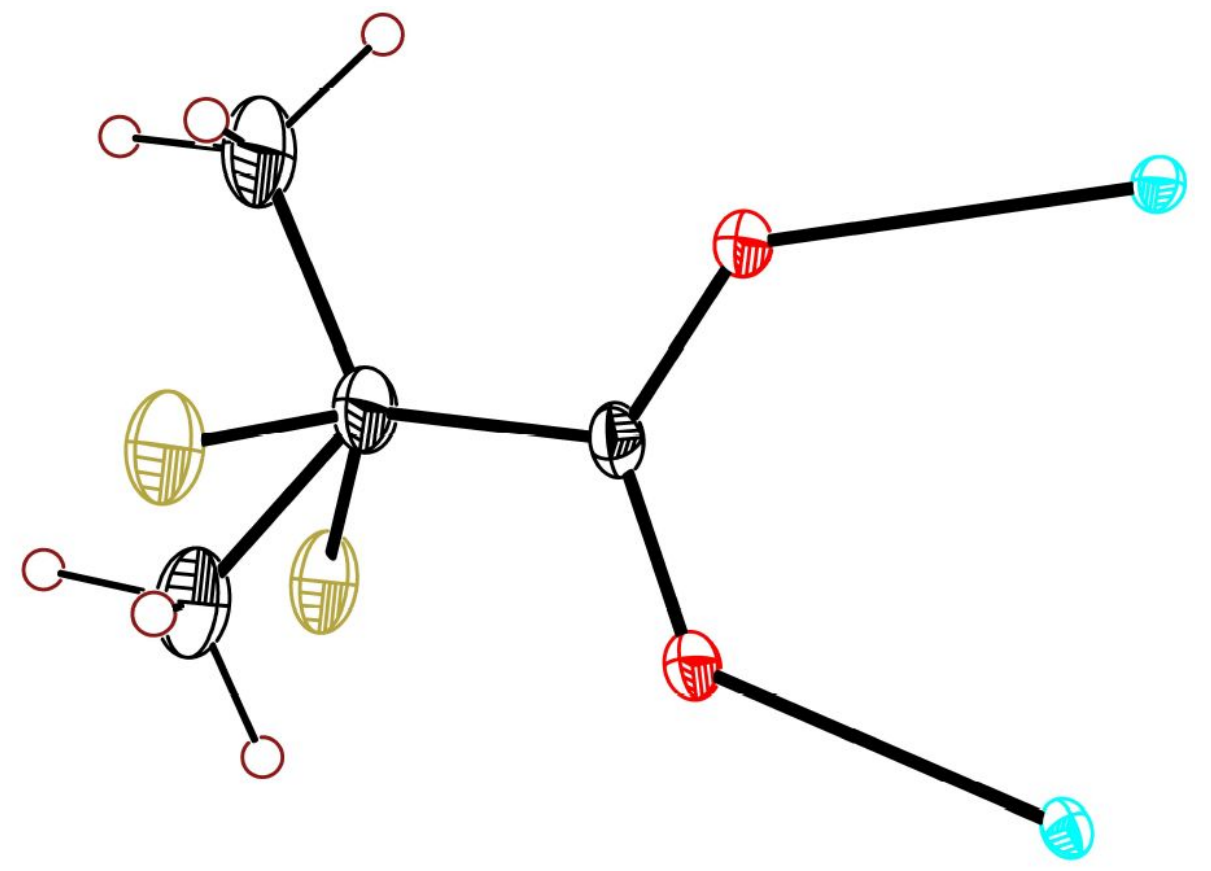

Figure S19. ORTEP figure ${ }^{[3]}$ highlighting the disorder of the $-\mathrm{CH}\left(\mathrm{CH}_{3}\right)_{2}$ moiety of $\mu_{2}-\eta_{1}: \eta_{1}$ $\mathrm{Zr} \cdots \mathrm{Zr}$-bridging isobutyrate ligands in $\mathbf{2}$. The refinement of two possible positions using a combination of PART and EADP commands yielded 0.74 site occupancy for the $\mathrm{C}$ atoms of the two methyl groups shown in black and 0.26 for the $\mathrm{C}$ positions highlighted as light-brown ellipsoids. H atoms (brown balls) were located only of the positions with higher site occupancy. SIMU restrictions were applied to refine $\mathrm{C}$ atoms of the disordered methyl groups as well as the $\mathrm{C}$ atom of the $\mathrm{CH}$ group to which they are bound. Color code for other thermal ellipsoids: Zr cyan, O red, C black. 


\section{References}

[1] Brown, I. D; Altermatt, D. Bond-Valence Parameters Obtained from a Systematic Analysis of the Inorganic Crystal Structure Database. Acta Crystallogr. 1985, B41, 244-247.

[2] Knížek, K. Kalvados - Software for crystal structure and powder diffraction; see http://www.fzu.cz/ knizek/kalvados/index.html)

[3] Burnett, M. N.; Johnson, C. K. ORTEP-III: Oak Ridge Thermal Ellipsoid Plot Program for Crystal Structure Illustrations, Oak Ridge National Laboratory Report ORNL6895, 1996. 